\title{
Aortic stent graft leak and aneurysm rupture after alteplase for stroke
}

\author{
Michael Mayette MD, Charles St-Arnaud MD
}

Cite as: CMAJ 2019 June 24;191:E709-11. doi: 10.1503/cmaj.181698

A n 81-year-old man presented to our hospital with signs and symptoms suggestive of a left middle cerebral artery stroke. The patient had a history of stable coronary artery disease, ischemic cardiomyopathy with a left ventricular ejection fraction of $45 \%$, well-controlled hypertension, hyperlipidemia and an abdominal aortic aneurysm for which he had undergone an endovascular aortic repair procedure with a prosthesis (SETALatecba balloon-expandable stent graft) 10 months earlier. The SETA prosthesis is balloon expandable, rather than the selfexpandable prosthesis that is most commonly used. Three months after his endovascular aortic repair procedure, the patient had undergone a routine, follow-up, contrast-enhanced abdominal computed tomography (CT) scan, which showed no sign of prosthesis malposition, malfunction or endoleak. The patient had no known kidney or liver dysfunction. The patient's medications included acetylsalicylic acid $80 \mathrm{mg}$ daily, metoprolol $25 \mathrm{mg}$ twice daily, rosuvastatin $40 \mathrm{mg}$ daily and ramipril $10 \mathrm{mg}$ daily.

On the day of admission, the patient presented with persistent right-sided hemiparesis and aphasia with a calculated National Institutes of Health stroke scale of 12 (www.ninds.nih. gov/sites/default/files/NIH_Stroke_Scale_Booklet.pdf; this scale ranges from 0 to 42, with higher scores indicating more severe neurologic deficits; although cases must be evaluated individually, scores $0-5$ are often considered mild, 6-14 are moderate, and scores $>14$ are considered severe). A brain CT scan showed cortical and subcortical hypodensities in the left precentral gyrus but no hemorrhagic stroke. An angio CT scan showed no proximal thrombus, precluding the option for catheter-guided thrombectomy. After careful review of contraindications and obtaining informed consent from the patient's substitute decision-maker (his spouse), we began systemic thrombolysis 2 hours and 50 minutes after the patient was last seen normal. We administered alteplase $0.9 \mathrm{mg} / \mathrm{kg}$ as per our local protocol (10\% as bolus, $90 \%$ as 60 -minute infusion). The patient's blood pressure at the start of the treatment was $142 / 67 \mathrm{~mm} \mathrm{Hg}$.

Although the patient's neurologic deficits began to improve within 1 hour of beginning treatment, we noted substantial arterial hypotension starting 90 minutes after the initial bolus of alteplase. Hypotension rapidly progressed to shock with blood pressure below $70 / 35 \mathrm{~mm} \mathrm{Hg}$, despite fluid resuscitation and administration of vasopressors. The patient developed rapidly progressing abdominal distension and pain. With ongoing

\section{KEY POINTS}

- Endoleak is a frequent complication after endovascular aortic repair and is a risk factor for spontaneous rupture of aneurysm.

- Administration of thrombolytics in patients with known abdominal aortic aneurysm or endovascular aortic repair, even remotely installed, is associated with endoleak or aneurysm rupture, and may represent a relative contraindication.

- Patients known with aortic aneurysm or endovascular aortic repair should be carefully evaluated and be informed about the risk of catastrophic bleeding before administration of thrombolytic drugs.

resuscitation, he underwent an abdominal CT scan showing active bleeding originating from the right iliac limb of the aortic stent graft, bleeding into a ruptured aneurysmal sac and into the abdomen (type III endoleak) (Figure 1).

The patient underwent urgent endovascular control of the hemorrhage with an occlusive balloon in the right iliac artery, followed by definitive treatment with a percutaneous Zenith stent graft (Cook Medical). Aortoiliac angiogram confirmed control of the leak. This was followed by abdominal decompression with laparotomy and drainage of more than $2 \mathrm{~L}$ of blood from the peritoneal space. He left the operating room with an open abdomen and vacuum-assisted closure dressing. He had coagulopathy, hypothermia from massive transfusions, lactic acidosis and acute anuric renal failure. Later that night, the immediate family decided to proceed to comfort care and withdrawal of lifesustaining measures, considering the patient's age, comorbidities and previously expressed wishes.

We reported this adverse event after administration of alteplase in the Canada Vigilance Adverse Reaction Online Database.

\section{Discussion}

Endovascular therapies for the management of infrarenal abdominal aortic aneurysms are increasingly performed because of their favourable early safety profile, shorter hospital stays and decreased short-term mortality, when compared with open surgical repair. ${ }^{1,2}$ However, long-term outcomes remain generally more favourable with open repair because of late complications associated with endovascular prosthesis., ${ }^{1,2}$ 


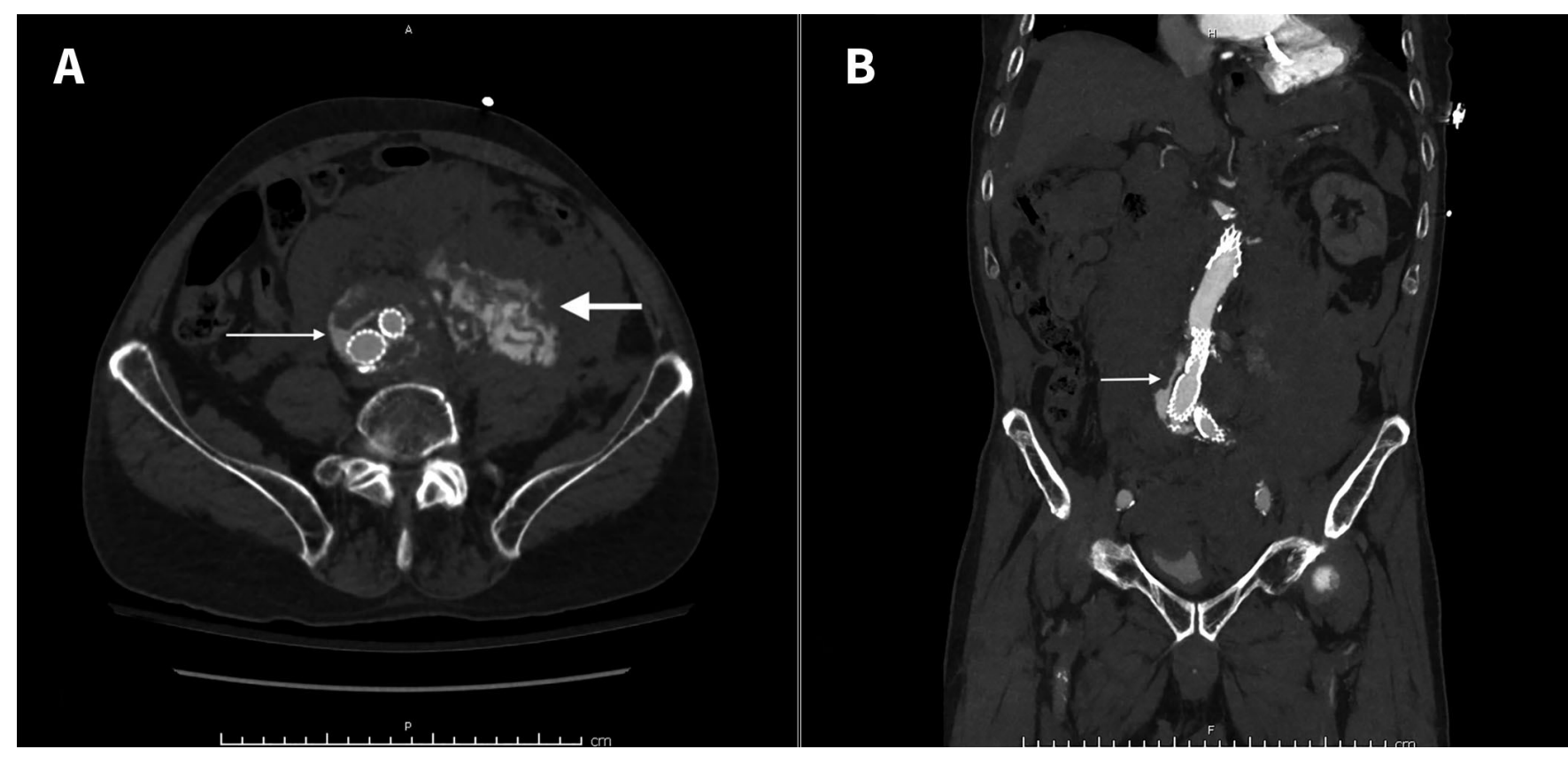

Figure 1: Axial (A) and coronal (B) contrast-enhanced computed tomography scan of the abdomen of an 81-year-old man, showing active bleeding (thin arrow) originating from the middle section of the aortic graft (type III endoleak [Box 1]) with associated intra-abdominal hematoma (thick arrow).

The most important late complications are leaks, which have been described in $30.5 \%$ of patients after endovascular aortic repair procedures. ${ }^{3}$ Endoleaks are classified as 5 different types of endoleaks and endotension (Box 1). Endotension, sometimes referred to as type $V$ endoleak, consists of increased expansion of the aneurysm sac with growth surrounding the stent graft but no actual leak identified. In most patients, endoleaks and endotension will be discovered on radiologic follow-up, while some will present with subacute progression of abdominal pain. Prompt radiologic evaluation will allow for timely intervention before aneurysm rupture. In a cohort of 39966 Medicare beneficiaries who underwent endovascular aortic repair, aneurysm rupture occurred in 5.4\% of patients over an 8-year follow-up. ${ }^{4}$ Risk factors for aneurysm rupture after endovascular aortic repair are the presence of an endoleak, stent migration and, potentially, the size of the underlying aneurysm. ${ }^{5}$ Aneurysm rupture occurs at a constant rate after endovascular aortic repair ${ }^{4}$ and delayed length of time since the original repair should not preclude the possibility of aneurysm rupture.
Thrombolytics are used frequently for various indications, including strokes, massive pulmonary embolism and limb ischemia. Multiple absolute and relative contraindications exist and must be systematically reviewed before those drugs are administered. Our case highlights a potential complication of the use of thrombolytics with aortic endovascular stent grafts, even when inserted months to years earlier (10 mo in our patient; even longer in other case reports).

Appendix 1 (available at at www.cmaj.ca/lookup/suppl/ doi:10.1503/cmaj.181698/-/DC1) summarizes some similar cases we identified in the medical literature, in which patients with aortic endovascular stent grafts developed acute endoleaks after administration of thrombolytic agents (altepase or urokinase) for stroke or lower-extremity limb ischemia, some requiring endovascular or surgical repair procedures. Of note, out of 178 reported adverse events associated with alteplase (most of those being intracerebral hemorrhagic complications or allergic reactions) in the Canada Vigilance Adverse Reaction Online Database (www.canada.ca/en/health-canada/services/drugs-health -products/medeffect-canada/adverse-reaction-database.html),

Box 1: Subtypes of endoleaks

\begin{tabular}{|lll}
\hline Type of endoleak & \multicolumn{1}{c}{ Definition } & Characteristics and management \\
\hline Type I & Leak of graft ends (inadequate seal) & High pressure, high risk. Requires urgent reintervention \\
\hline Type II & Sac filling via branch vessel & Low pressure. Intervention on branch vessel may be required depending on sac growth \\
\hline Type III & Leak via defect in graft fabric & High pressure, high risk. Requires urgent reintervention \\
\hline Type IV & Porous graft & Most frequently self-limited and requires no treatment \\
\hline Type V & Endotension & Incompletely understood pathophysiology and difficult diagnosis. Treatment \\
\hline
\end{tabular}

Adapted from: White GH, Yu W, May J, et al. Endoleak as a complication of endoluminal grafting of abdominal aortic aneurysms: classification, incidence, diagnosis and management. J Endovascular Surg 1997;4:152-168. 
only 3 are listed as intra-abdominal or peritoneal hemorrhage and none mention the presence of a stent graft. It has been proposed that stent grafts become more porous after exposure to thrombolytics, but dynamic changes in the integrity of wall thrombus may also play a role. ${ }^{6}$ The formation of normal wall thrombus after graft implantation is part of the graft's integrity and impermeability, especially for type IV endoleak. It is plausible that thrombolytics may disrupt the integrity of normal wall thrombus and precipitate formation of new leaks. Systemic anticoagulation with warfarin, which causes less disruption of clot homeostasis than thrombolytics, has been shown to increase the risk of formation of all types of endoleak. ${ }^{7}$ However, it is unclear whether thrombolytics cause new leaks to form or if leaks are simply undiagnosed before administration of the agent.

In unrepaired aortic aneurysm, use of thrombolytics has been associated with leak of the aneurysm ${ }^{8}$ and dislodgement of thrombus with distal embolization. ${ }^{9}$ These reports suggest that unruptured arterial aneurysms maintain a fragile homeostasis from the balance of coagulation and innate fibrinolysis inside the mural thrombus. This homeostasis has been extensively studied, ${ }^{10-11}$ but the impacts of thrombolytic drugs could be critical for patients with underlying aneurysmal vascular disease.

Current guidelines, including the American Heart Association/ American Stroke Association ${ }^{12}$ and Canadian guidelines, ${ }^{13}$ do not include the presence of an aortic aneurysm or a prior aortic stent graft as a consideration before thrombolysis. As endoleaks are both common and associated with aneurysmal rupture after endovascular aortic repair, we believe those should be listed as relative contraindications for administration of thrombolytics, whether for stroke, myocardial infarction, pulmonary embolism or limb ischemia. If time permits, we suggest that patients with a known history of endovascular aortic repair undergo abdominal CT angiogram before administration of thrombolytics agents, to help identify patients at higher risk of aneurysm rupture. Because the absence of an endoleak does not preclude aneurysm rupture, we suggest that risks associated with administration of thrombolytics in the presence of an aortic aneurysm or stent graft be presented to the patient or substitute decision-maker when discussing the risks and benefits of this therapy. Balancing these risks and benefits is challenging, as the actual risk of rupture is unknown; we can assume the risk remains low, as only a few case reports of this complication have been published. This risk, however small, may help with making the decision in ambiguous cases (for example, stroke with minor deficits, or submassive pulmonary embolism). When evaluating risk and benefits, we suggest that the time delay between graft installation and presentation be noted, careful review of follow-up imaging be undertaken and, when in doubt, consultation with a vascular surgeon before administration be considered.

\section{Conclusion}

Aneurysm rupture or endovascular endoleak after administration of thrombolytic agents is an uncommon complication. However, this complication may be catastrophic, as in our case. Most hospitals use preprinted protocols for thrombolytics with contraindications listed as a reminder for clinicians. We believe the pres- ence of an untreated aortic aneurysm or an aortic stent graft should be added to this list as an important consideration or relative contraindication.

\section{References}

1. United Kingdom EVAR Trial Investigators; Greenhalgh RM, Brown LC, Powell JT, et al. Endovascular versus open repair of abdominal aortic aneurysm. $N$ Engl J Med 2010;362:1863-71.

2. Patel R, Sweeting MJ, Powell JT, et al.; EVAR Trial Investigators. Endovascular versus open repair of abdominal aortic aneurysm in 15-years' follow-up of the UK endovascular aneurysm repair trial 1 (EVAR trial 1): a randomised controlled trial. Lancet 2016;388:2366-74.

3. Lal BK, Zhou W, Li Z, et al.; OVER Veterans Affairs Cooperative Study Group. Predictors and outcomes of endoleaks in the Veterans Affairs Open Versus Endovascular Repair (OVER) trial of abdominal aortic aneurysms. J Vasc Surg 2015;62:1394-404.

4. Schermerhorn ML, Buck DB, O'malley AJ, et al. Long-term outcomes of abdominal aortic aneurysm in the Medicare population. N Engl J Med 2015;373:328-38.

5. Wyss TR, Brown LC, Powell JT, et al. Rate and predictability of graft rupture after endovascular and open abdominal aortic aneurysm repair: data from the EVAR Trials. Ann Surg 2010;252:805-12.

6. Belchos J, Wheatcroft M, Prabhudesai V, et al. Development of endotension after multiple rounds of thrombolysis after endovascular aneurysm repair. J Vasc Surg Cases 2015;1:24-7.

7. Lazarides MK, Georgiadis GS, Charalampidis DG, et al. Impact of long-term warfarin treatment on EVAR durability: a meta-analysis. J Endovasc Ther 2014; 21:148-53.

8. Hale PC, Smith SM, Taylor PR. Aortic aneurysm leak after streptokinase treatment for myocardial infarction. Eur J Vasc Surg 1993;7:738-9.

9. de Melo Bernardi FL, Rezende PC, Garzillo CL, et al. Massive thromboembolism from an abdominal aortic aneurysm after successful thrombolysis for an acute myocardial infarction. Case Rep Clin Med 2015;4:45-9.

10. Houard X, Rouzet F, Touat Z, et al. Topology of the fibrinolytic system within the mural thrombus of human abdominal aortic aneurysms. J Pathol 2007;212:20-8.

11. Skagius E, Siegbahn A, Bergqvist D, et al. Fibrinolysis in patients with an abdominal aortic aneurysm with special emphasis on rupture and shock. $J$ Thromb Haemost 2008;6:147-50.

12. Powers WJ, Rabinstein AA, Ackerson T, et al. 2018 guidelines for the early management of patients with acute ischemic stroke: a guideline for healthcare professionals from the American Heart Association/American Stroke Association. Stroke 2018;49:e46-110.

13. Boulanger JM, Lindsay MP, Gubitz G, et al. Canadian Stroke best practice recommendations for acute stroke management: prehospital, emergency department, and acute inpatient stroke care, 6th edition, update 2018. Int J Stroke 2018; 13:949-84.

\section{Competing interests: None declared.}

This article has been peer reviewed.

The authors have obtained consent from the patient's family.

Affiliation: Department of Medicine, Internal Medicine and Critical Care Division (Mayette, St-Arnaud), Université de Sherbrooke, Sherbrooke, Que.

Contributors: Both authors contributed to the conception and design of the work, drafted the manuscript, revised it critically for important intellectual content, gave final approval of the version to be published and agreed to be accountable for all aspects of the work.

Acknowledgements: The authors thank Dr. Marc-Antoine Despatis, Dr. Véronique Lapie (both from the Department of Surgery, Vascular Surgery division, Centre Hospitalier Universitaire de Sherbrooke) and Dr. François Evoy (from the Department of Medicine, Neurology division, Centre Hospitalier Universitaire de Sherbrooke) for their contribution to the manuscript.

Correspondence to: Michael Mayette, michael.mayette@usherbrooke.ca 